\title{
STUDY OF MISFIT DISLOCATIONS PROFILES IN ZnSe/GaAs STRUCTURES BY RAMAN SCATTERING*
}

\author{
W. BALA \\ Institute of Physics, N. Copernicus University, Grudziądzka 5/7, 87-100 Toruń, Poland \\ M. Drozdowski and M. KozIELSKI \\ Institute of Physics, Poznań Technical University, Piotrowo 3, 60-965 Poznań, Poland
}

In this paper we present the Raman scattering measurements of the ZnSe epilayers grown on (001) GaAs substrate by molecular beam epitaxy method. We have studied dependence of the frequency shift of $\mathrm{LO}(\mathrm{ZnSe})$ mode in the Raman spectra vs. thickness of the ZnSe layer. The intensity of $\mathrm{LO}(\mathrm{ZnSe}) / \mathrm{LO}(\mathrm{GaAs})$ ratio vs. orientation angle $\alpha$ of the $\boldsymbol{E}$ vector of the exciting light on the $\mathrm{ZnSe} / \mathrm{GaAs}$ interface relatively to the sample orientation is presented too.

PACS numbers: $78.30 . \mathrm{Hv}$

\section{Introduction}

In the last years many research efforts have been addressed to the study of wide gap AII-BVI heterostructures derived from $\mathrm{ZnSe}$ because of their potential optoelectronic applications in the blue-green spectral region [1]. The presence of a large number of defects at the $\mathrm{ZnSe} / \mathrm{GaAs}$ interface deteriorates the electronic properties and the performance of these devices. Because the optical and electrical properties of interface are strongly degraded by the presence of misfit dislocations, it is important to estimate the critical thickness of the epilayer and profile of the dislocations at interface [2].

In this paper we present the Raman scattering measurements of the $\mathrm{ZnSe}$ epilayer with different thickness grown on GaAs substrate. The dependence of the frequency shift of $\mathrm{LO}(\mathrm{ZnSe})$ mode vs. thickness of $\mathrm{ZnSe}$ epilayer is presented. Dependence of the relative intensity $\mathrm{LO}(\mathrm{ZnSe}) / \mathrm{LO}(\mathrm{GaAs})$ mode as a function of the orientation angle $\alpha$ of the sample and the $\boldsymbol{E}$ vector of exciting light is also presented. From these results we can estimate an array of misfit dislocations created during the growth of the $\mathrm{ZnSe}$ epilayer.

*This work was supported in part by the Committee for Scientific Research under grand No. 223209102 (Poznań) and grand No. 203299101 (Toruń). 


\section{Experimental}

The ZnSe epilayers were grown at $595 \mathrm{~K}$ on (001) GaAs substrate from separated sources with $6 \mathrm{~N} \mathrm{Zn}$ and $6 \mathrm{~N}$ Se elements [3]. The growth rates are in the range $0.4-0.8 \mu \mathrm{m} / \mathrm{h}$. The Se/Zn beam pressure ratio of about 2 was employed.

The sample was mounted in the cryostat with the $\mathrm{ZnSe} / \mathrm{GaAs}$ interface oriented parallel to the $\boldsymbol{E}$ vector of the exciting light and perpendicular to the axis of the collecting light. The Raman polarized spectra of the epilayers were performed using quasi-back scattering geometry described elsewhere [4]. An Ar ion laser operating at $458 \mathrm{~nm}$ was used to excite the Raman spectra. The relatively large absorption coefficient of $\mathrm{ZnSe}$ material at room temperature assured us that the incident light was penetrating only the upper part of the $\mathrm{ZnSe}$ film when samples with different thickneses were investigated. In case when the dependence of $\mathrm{LO}(\mathrm{ZnSe}) / \mathrm{LO}(\mathrm{GaAs})$ ratio as a function of the relative orientation angle $\alpha$ of the $\boldsymbol{E}$ vector of the incident light vs. orientation of the interface of $\mathrm{ZnSe} / \mathrm{GaAs}$ heterojunction was investigated the rotating system was employed.

\section{Results and discussion}

Representative results of $\mathrm{LO}(\mathrm{ZnSe}) / \mathrm{LO}(\mathrm{GaAs})$ ratio vs. orientation angle $\alpha$ for the samples with thickneses equal to $0.1 \mu \mathrm{m}$ and $0.3 \mu \mathrm{m}$ are presented in Fig. 1 (curve $a$ and $b$, respectively). As can be seen from Fig. 1, the ratio of LO modes shows a periodic change vs. orientation angle $\alpha$. This is probably caused by misfit

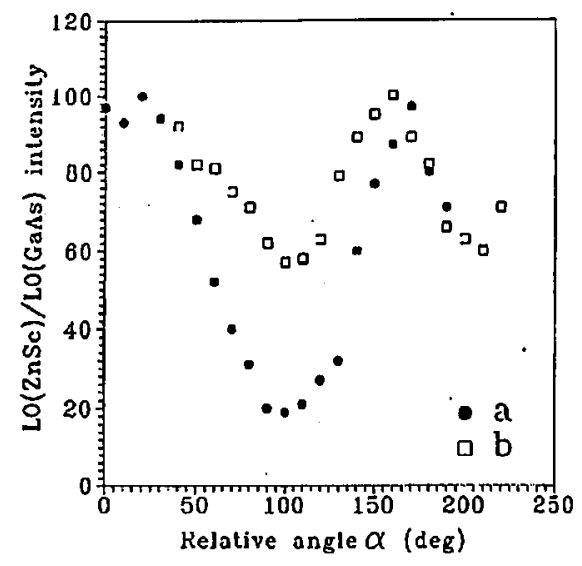

Fig. 1. Dependence of $\mathrm{LO}(\mathrm{ZnSe}) / \mathrm{LO}(\mathrm{GaAs})$ ratio as a function of the relative orientation angle $\alpha$ of the $\boldsymbol{E}$ vector of the incident light on the interface of $\mathrm{ZnSe} / \mathrm{GaAs}$ heterojunction for different ZnSe epilayer thickness: $a=0.1 \mu \mathrm{m}, b=0.3 \mu \mathrm{m}$.

dislocations which are the Lomer dislocations with $1 / 2\langle 100\rangle$ Burgers vector lying in the plain of interface, described elastically as the Volterra dislocations $[2,4,5]$. For coherent growth $(h<0.08 \mu \mathrm{m})$ we did not observe the periodic change of 
$\mathrm{LO}(\mathrm{ZnSe}) / \mathrm{LO}(\mathrm{GaAs})$ ratio vs. orientation angle $\alpha$. The ratio of $\mathrm{LO}$ modes for a given thickness was constant.

The dependence of the frequency shift of $\mathrm{LO}(\mathrm{ZnSe})$ mode in the Raman spectra vs. thickness of the $\mathrm{ZnSe}$ epilayer is presented in Fig. 2. This dependence

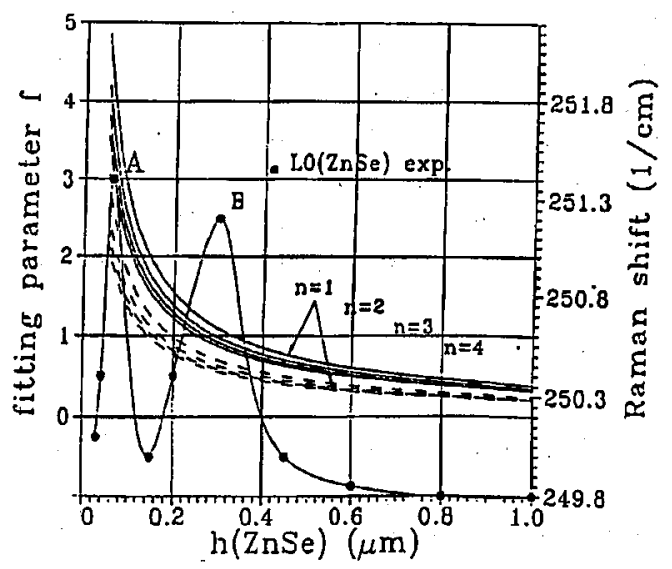

Fig. 2. The frequency shift of the $\mathrm{LO}(\mathrm{ZnSe}$ ) mode vs. thickness of $\mathrm{ZnSe}$ epilayer (full points). The theoretical calculation of parameter $f$ proportional to the critical thickness $h$ for different $n$ according to Eq. (1) - (dashed line) and Eq. (2) - (solid line).

exhibits two maxima [6]. For the first step of growing, when the thin epilayer is formed coherently, we have large elastic strain in the interface plane. In this case the frequency of $\mathrm{LO}(\mathrm{ZnSe})$ mode is shifted to higher energies and for the thickness equal to $0.08 \mathrm{~mm}$ we observe the first maximum (Fig. 2, peak $A$ ). For the larger thicknesses $(h>0.08 \mu \mathrm{m})$ the LO mode shifts to lower energies. For the sample with the ZnSe epilayers thickness equal to $0.30 \mathrm{~mm}$ we observe another maximum (Fig. 2, peak $B$ ). For the sample with the $\mathrm{ZnSe}$ epilayer thicker than $0.6 \mu \mathrm{m}$ $\mathrm{LO}(\mathrm{ZnSe})$ mode has a tendency to approach the frequency of stress-free bulk crystals. From this feature we can experimentally estimate the critical thickness of the epilayer $h$ and the profile of the dislocations.

The concept of misfit dislocation into a strained layer treated in the Volterra dislocation model was recently introduced by Hirth and Feng [2]. In this paper the authors propose two different critical thickneses: the critical thickness $\left(h_{0}\right)$ referring to the layer thickness to form the first stable dislocation pair in a strained-layer structure $[7,8]$. and the critical thickness $\left(h_{c}\right)$ for other arrangements of dislocations, including that for complete relaxation of misfit by an array of misfit dislocations [5]. In observations of dislocations in $\{001\}$ later structures, the equilibrium Lomer array is found only for very large misfits or for $h \gg h_{\mathrm{c}}[5,9,10]$. Instead, $1 / 2\langle 110\rangle$ dislocations form on inclined $\{111\}$ planes and glide to deposit on interface. These dislocations are less effective in removing misfit because the misfit. component is only one-half the magnitude of the Burgers vector of the Lomer dislocations. In this case we have the critical thickness $\left(h_{0}\right)$ for which an epilayer 
grows without misfit dislocations $[2,5,11]$ :

$$
h_{0}=\frac{b(4-\nu)}{4 \pi(1+\nu) \varepsilon_{0} f} \ln \left(\frac{\sqrt{3}}{\sqrt{2} b n} h_{0}\right)
$$

where $b$ is magnitude of the Burgers vector, $\nu$ - the Poissons ratio, $\varepsilon_{0}$ - absolute value of the total misfit, $f$ - the fitting parameter and $n$ - the multiple of $b$ [11].

For heterostructures, People and Bean [12] have assumed that interfacial misfit dislocations are generated when the areal strain energy density exceeds the self-energy of an isolated dislocation of a given type: screw, edge, and half-loop dislocations. The maximum misfit at which the dislocation free interface can be formed takes place when the total misfit is equal to the minimum elastic strain energy. In this case the critical thickness $h_{\mathrm{c}}$ may be written as

$$
h_{\mathrm{c}}=\frac{b}{\pi \varepsilon_{0} f} \ln \left(\frac{\sqrt{2}}{b n} h_{\mathrm{c}}\right) \text {. }
$$

In Fig. 2 we also present the theoretical parameter $f$ proportional to the critical thickness $h_{0}$ (dashed line) and $h_{\mathrm{c}}$ (solid line) obtained for two cases described by Eqs. (1) and (2), respectively. As can be seen from Fig. 2 the most energetically favourable situation to create the Lomer dislocations is for $f=1$ (peak $B$ ) and inclined dislocations is for $f=2$ (peak $A$ ).

\section{References}

[1] G. Kudlek, J. Gutowski, J. Lumin. 52, 55 (1992).

[2] X. Feng, J.P. Hirth, J. Appl. Phys. 72, 1386 (1992).

[3] W. Bała, F. Firszt, Proc. 3rd Conf. Surf. Phys., Zakopane, 1988 V, 27 (1989).

[4] M. Kozielski, M. Drozdowski, P. Ziobrowski, W. Bała, SPIE - The International Sociely for Optical Engineering, in press.

[5] J.P. Hirth, X. Feng, J. Appl. Phys. 67, 3343 (1990).

[6] W. Bała, M. Drozdowski, M. Kozielski, Phys. Status Solidi A 130, K195 (1992).

[7] F.C. Frank, J.H. van der Merwe, Proc. R. Soc. Lond. A 198, 205 (1949); 198, 216 (1949).

[8] J.W. Matthews, in: Dislocations in Solids, Vol. 2, Ed. F.N. Nabarro, North-Holland, Amsterdam 1979, p. 461.

[9] R. Hull, J.C. Beam, J. Vac. Sci. Technol. A 7, 2580 (1989).

[10] J. Petruzzello, B.L. Greenberg, D.A. Cammack, R. Dalby, J. Appl. Phys. 63, 2299 (1988).

[11] E.P. O'Reilly, Semicond. Sci. Technol. 4, 121 (1989).

[12] R. People, J.C. Bean, Appl. Phys. Lett. 47, 322 (1985). 